\title{
Complete genomes and phylogenetic positions of bovine papillomavirus type 8 and a variant type from a European bison
}

\author{
Yoshimi Tomita · Ivan Literak · Tomoko Ogawa • \\ Zhongri Jin $\cdot$ Hiroshi Shirasawa
}

Published online: 16 November 2007

(C) Springer Science+Business Media, LLC 2007

Erratum to: Virus Genes (2007) 35(2): 243-249

DOI 10.1007/s11262-006-0055-y

Page 247, Fig. 4 "alignment of BPV-1" should read "alignment of OvPV-1, EEPV, RPV, DPV, BPV-1."
Page 248, Fig. 5 "BPV-1,-2,-3,-4,-5,-6, and" should read "BPV-1,-2,EEPV,-DPV,-RPV,-5 and", Fig. 6 "of EEPV," should read "of OvPV-1 EEPV."

The online version of the original article can be found under doi:10.1007/s11262-006-0055-y.

Y. Tomita $(\bowtie) \cdot$ Z. Jin $\cdot$ H. Shirasawa

Department of Molecular Virology, Graduate School of

Medicine, Chiba University, 1-8-1 Inohana, Chuou-ku,

Chiba 260-8670, Japan

e-mail: tomita@faculty.chiba-u.jp

H. Shirasawa

e-mail: sirasawa@faculty.chiba-u.jp

I. Literak

Department of Biology and Wildlife Diseases, Faculty of Veterinary Hygiene and Ecology, University of Veterinary and Pharmaceutical Sciences, Palackeho 1-3, 62142 Brno, Czech Republic

e-mail: literaki@vfu.cz

T. Ogawa

Division of Virology, Chiba Prefectural Institute of Public Health, 666-2 Nitona-cho, Chuou-ku, Chiba 260-8715, Japan

e-mail: t.ogw11@mb.pref.chiba.jp 